
\title{
25 Research Soure \\ Long-Range Transport of Sulfur Dioxide Emissions From External Sources to Tehran
}

\section{Reza Bashiri Khuzestani}

Shahid Beheshti University

Ahmad Taheri

Tehran Air Quality Control Company

Bijan Yeganeh ( $\sim$ b_yeganeh@sbu.ac.ir)

Shahid Beheshti University

\section{Research Article}

Keywords: Sulfur Dioxide (SO2), Long-range regional transport, local emission, remote sensing, population exposures, Tehran

Posted Date: December 28th, 2021

DOI: https://doi.org/10.21203/rs.3.rs-1158752/v1

License: (c) (i) This work is licensed under a Creative Commons Attribution 4.0 International License. Read Full License 


\section{Abstract}

Large-scale emissions of sulfur dioxide $\left(\mathrm{SO}_{2}\right)$ from the combustion of heavy fuel oils are deteriorating the air quality in Tehran and regularly causing complex atmospheric pollution situations and human health concerns. Our analysis of the long-term $\mathrm{SO}_{2}$ emission data in Tehran confirmed that the magnitude of local $\mathrm{SO}_{2}$ emission sources is not adequate to reach $\mathrm{SO}_{2}$ concentrations to their present levels. Tehran is predominantly affected by regional transport of $\mathrm{SO}_{2}$ from exterior sources further away located in Iraq, Saudi Arabia, and adjacent provinces neighboring Tehran. Approximately $80 \%$ of total $\mathrm{SO}_{2}$ emissions in Tehran were observed to have impacts from the external hotspots outside of Tehran. While local emission sources only contribute around $20 \%$ of the total $\mathrm{SO}_{2}$ emissions. Bivariate polar plots, k-mean cluster, pairwise polar correlation, and PSCF analysis provided evidence for the impact of large-scale transport of $\mathrm{SO}_{2}$ emissions from external locations from the west/northwest, north/northeast, and south/southwestern areas of the region. Further observations of these hotspot areas observed in our analysis with TROPOMI satellite data confirmed significant $\mathrm{SO}_{2}$ emissions resulting from the consumption of heavy fuel oils in thermal power plants and oil/gas refineries. Overall, the results suggested that the regulatory strategies for controlling local traffic emissions of $\mathrm{SO}_{2}$ in Tehran would not be beneficial for reducing public health exposures to $\mathrm{SO}_{2}$ in Tehran. Such improvements can be attained mainly by diminishing the emission sources located further away from Tehran.

\section{Introduction}

High sulfur dioxide $\left(\mathrm{SO}_{2}\right)$ emission from burning heavy fuels oils in power plants and industries in Tehran and surrounding regions are deteriorating the air quality in that middle eastern megacity in recent years (Amini et al., 2014; Hassanzadeh et al., 2009; Nazari et al., 2012). $\mathrm{SO}_{2}$ is a highly reactive gas with the largest emission sources from fossil fuel combustion in power plants and industrial processes (Nazari et al., 2012). Smaller shares of $\mathrm{SO}_{2}$ emissions would correspond to roadway traffic sources and high sulfur content fuels in industries and non-traffic transportations (Nazari et al., 2012). According to the latest emission inventories of Tehran, $\mathrm{SO}_{2}$ emissions are estimated at around 13,000 tons per year, with $94 \%$ of its contributions being related to stationary sources, while mobile sources only contribute to $6 \%$ of total $\mathrm{SO}_{2}$ emission (Shahbazi et al., 2019). The reports of fuel quality from Tehran Air Quality Control Company (AQCC) showed that the sulfur content of diesel fuel consumed by mobile and roadway sources decreased significantly by more than $98 \%$ since the end of 2016 (Sulfur content decreased from $4000 \mathrm{ppm}$ to around $75 \mathrm{ppm}$ ) causing substantial improvements in the production and emissions of $\mathrm{SO}_{2}$ from mobile sources (Ghadiri et al., 2017; Shahbazi et al., 2019). However, despite the reduction in $\mathrm{SO}_{2}$ emissions of mobile sources, large amounts of $\mathrm{SO}_{2}$ were released into the atmosphere, mainly from uncontrolled stationary sources in Tehran and its surrounding regions. Recent studies by NASA OMI satellites capturing more than 500 major point sources of $\mathrm{SO}_{2}$ emissions across the globe confirmed Iran as one of the greatest anthropogenic $\mathrm{SO}_{2}$ emission hotspots in the world (Dahiya and Myllyvirta, 2019; Zhong et al., 2020). The increased use of coal combustion and heavy fuel oil for increasing energy 
production demands in the area, partly due to a lack of an effective flue gas desulfurization (FGD) process and slow implementation of emission standards, raises concern about complex air quality situations in the region. (Nazari et al., 2012).

In some extreme cases, unpleasant odors were experienced in large areas of Tehran, which were later understood to be linked to high $\mathrm{SO}_{2}$ production and emission transportation in Tehran. Our analysis during the pollution episodes in Tehran confirmed extremely high peaks of $\mathrm{SO}_{2}$ concentrations lasting for a relatively long period were observed in large areas of the city, indicating the persistence of strong sources of $\mathrm{SO}_{2}$ in the city and the surrounding areas.

To date, emission regulations and monitoring analysis were far too weak to sufficiently evaluate and quantify $\mathrm{SO}_{2}$ productions and their high emitting hotspot sources in the area. Source apportionment analysis in Tehran showed that sulfate could take up to $40 \%$ of total $\mathrm{PM}_{2.5}$ contributions in the city, most of which were due to high primary $\mathrm{SO}_{2}$ emission sources (Esmaeilirad et al., 2020). $\mathrm{SO}_{2}$ has remained a critical air quality concern in Tehran; therefore, a comprehensive investigation of the extent of local and regional transport of $\mathrm{SO}_{2}$, including their hotspot emission sources, is crucial for policymaking and developing future air pollution mitigation plans in Tehran.

To the best of our knowledge, comprehensive analyses on $\mathrm{SO}_{2}$ emission and their local and regional hotspot sources are still poorly constrained to be sufficiently comprehensive and quantifiable. Chemical transport models cannot provide a reliable estimate of $\mathrm{SO}_{2}$ concentration due to the lack of a proper emission inventory in the study area. In addition, accessing such a long-distance $\mathrm{SO}_{2}$ emission database was impossible for the regions outside Tehran. Therefore, remote sensing observations and the spatial distributions of columnar $\mathrm{SO}_{2}$ over Iran and its surrounding area were used for the whole study period. Furthermore, a series of source apportionment analyses were conducted, and $\mathrm{SO}_{2}$ profiles were closely evaluated at various regions in Tehran to quantify long-term regional-scale contributions using air quality and long-term $\mathrm{SO}_{2}$ monitoring networks. Our analysis will clarify how long-range regional transport of $\mathrm{SO}_{2}$ and the different local emission sources and spatial-scale contributions will govern $\mathrm{SO}_{2}$ pollution in the region.

\section{Materials And Methods 2.1. Study area}

Located in an urban region including 15 million inhabitants in its larger municipal areas, Tehran is considered Iran largest and one of the most populous cities in the world (Bayat et al., 2019; Yeganeh et al., 2021). Tehran's climate can feature a cold and semi-arid characteristic with a yearly mean temperature of $17^{\circ} \mathrm{C}$ and annual precipitation of about $230 \mathrm{~mm}$. The average annual highs will be $25^{\circ} \mathrm{C}$, and the annual lows averages at around $10^{\circ} \mathrm{C}$. Extreme temperatures reaching up to $40^{\circ} \mathrm{C}$ could also be seen during July, while minimum temperatures of up to $-10^{\circ} \mathrm{C}$ could also happen during January. 
Comprehensive information on the time series of meteorological data is provided in Fig. S1 in the supporting information.

\section{2. $\mathrm{SO}_{2}$ monitoring}

Tehran has a network of 35 air quality monitoring stations mainly administered by the Department of Environment (DOE) and the Tehran AQCC. The stations belonging to AQCC were equipped with regular gas and $\mathrm{PM}$ analyzers (e.g., $\mathrm{CO}, \mathrm{NO}, \mathrm{NO}_{2}, \mathrm{NO}_{\mathrm{x}}, \mathrm{SO}_{2}, \mathrm{O}_{3}, \mathrm{PM}_{2.5}$, and $\mathrm{PM}_{10}$ analyzers) operating continuously over the city (Yeganeh et al., 2021). $\mathrm{SO}_{2}$ measurements data conducted at several governmental centers and community districts within Tehran were designated to represent diverse environments in Tehran and were categorized as urban-residential (TARB area), traffic hotspots (SHAD and R21 areas), industrial (R20 area), and residential (AGD and PIR areas). UV fluorescence analyzers (Model Serinus 50, Ecotech, Australia and Model AF22, Environment S.A, France) were used to analyse $\mathrm{SO}_{2}$ in all of the monitoring networks in Tehran. The $\mathrm{SO}_{2}$ data at six representative areas at various administrative divisions in Tehran were selected. The available database includes $\mathrm{SO}_{2}$ emission data for the period 2020-2021. The location of the monitoring sites and detailed descriptions and codes can be found in Fig. S2 and Table S1 in the supporting information. The overall data quality for the study period in all of our monitoring areas was reported good. We obtained an average of approximately $96 \%$ of data coverage with less than $4 \%$ of data missing values. More information on the data quality of this study is provided in Table S2 in the supporting information. Maintenance and calibration of the analyzers are performed regularly according to BS EN 14212: 2012 standard.

\subsection{Bivariate Polar Plots (BPPs) and k-mean cluster analysis}

Bivariate polar plots (BPPs) could be created by apportioning wind speed, wind direction, and measurement data in different wind speed-direction intervals (Khuzestani et al., 2017). The $u$ and $v$ wind component variables will be obtained by deconvoluting the wind components according to

Eq. $1 u=\bar{u} \cdot \sin \left(\frac{2 \pi}{\theta}\right), v=\bar{u} \cdot \cos \left(\frac{2 \pi}{\theta}\right)$

Where $u$ is the hourly average wind speed, and $\theta$ is the wind direction in degrees. A Generalized Additive Model (GAM) framework has finally been applied to $u, v$, and concentration (C) data to generate a continuous surface to define the measurement levels as a function of the wind constituents to obtain valid source descriptions. GAMs can be expressed as shown in Eq. 2.

Eq. $2 \sqrt{C_{i}}=\beta_{0}+\sum_{j=1}^{n} S_{j}\left(X_{i j}\right)+e_{i}$

Where $C_{i}$ is the pollutant $i$ concentration, $\beta 0$ is the average of response sj (xij) is the smooth function of value $i$ of covariate $j, n$ is the entire amount of covariates, and ei is the residual $i$. For better model 
diagnostics and improved residual distributions, the concentration data $\mathrm{Ci}$ will be square root transformed. Analysis of a wide range of scenarios suggests that appropriate details of surface concentration distributions could be obtained by binning 10-degree intervals of wind direction and 30 wind speed intervals. This strategy could guarantee an active data reduction approach without sacrificing the reliability of the polar plot. Due to the significant variability in wind direction data, it is usually recommended to use this data from various months or years to create a more finely resolved and robust surface interpolation of BPPs (Carslaw et al., 2006; Westmoreland et al., 2007).

To further analyze the BPP's identified features regarding the potential source characteristics, we performed a k-mean cluster analysis. This strategy can obtain similar cluster features in BPPs based on the similarity or dissimilarity between the data points. Polar clusters can select interesting features in BPPs as clusters based on different wind components. The fundamentals of polar clusters are k-mean clustering the BPPs as follows: Initially, random k points representing the initial group centroids will be selected from the surface generated by BPPs. All the objects and points will then be allocated to the groups that have the closest centroid. The positions of the k-points will then be repeatedly recalculated until the centroids no longer move. k-mean cluster analysis is similar to the idea of clustering trajectories described in detail in Carslaw and Beevers (2013). However, wind components and concentration data rather than the air mass origins will be used to identify similar groups.

\subsection{TROPOspheric Monitoring Instrument (TROPOMI) data analysis}

TROPOMI was launched on October 13, 2017, by the Sentinel-5 Precursor (S5P) from northern Russia. The satellite orbits the Earth at $817 \mathrm{~km}$ and has a repetition cycle of 17 days (KNMI, 2017). The SP5 has four spectrometers, one covering the short-term infrared and the other three covering the ultraviolet-near infrared ranges. The satellite data processing could obtain high-resolution $\left(3.5 \times 7 \mathrm{~km}^{2}\right)$ daily coverage data of $\mathrm{O}_{3}, \mathrm{NO}_{2}, \mathrm{SO}_{2}, \mathrm{CO}, \mathrm{CH}_{4}, \mathrm{HCHO}$, cloud and aerosol properties. Detailed information on the methodology of $\mathrm{SO}_{2}$ data processing has been described elsewhere in $\mathrm{KNMI}, 2017$. We analyzed the spatial distributions of columnar $\mathrm{SO}_{2}$ over Iran and its surrounding region for the whole year of 2020 .

In summary, The OFFL/L3-SO ${ }_{2}$ offers high-resolution offline images of $\mathrm{SO}_{2}$ concentration levels.

With 1-day revisit time and the high spatial resolution ( $3 \times 5.5 \mathrm{~km})$, S5P/TROPOMI is able to provide sufficient details, including the recognition of minor $\mathrm{SO}_{2}$ plumes. For the $\mathrm{L}_{3}-\mathrm{SO}_{2}$ product, the absorbing aerosol index is computed with measurements at 340 and $380 \mathrm{~nm}$ wavelengths. The OFFL/L3 products for the ares within the product's bounding box were constructed using the harp convert tool. The areaaverage data for the pixels with different values for different times were then merged together into a greater mosaic containing sets of different tiles with orthorectified raster data.

\subsection{Potential Source Contribution Function (PSCF)}


The hybrid single-particle Lagrangian integrated trajectory model (HYSPLIT version 5) was implemented to calculate the hourly-interval 48-h backward trajectories arriving at heights of $500 \mathrm{~m}$ above ground level at the monitoring areas in Tehran using a vertical velocity model. The Global Data Assimilation System (GDAS) one-degree archive data combined measurements into a gridded space with observed data (Khuzestani et al., 2017). Fig. S3 in the supporting information illustrates the frequencies of the trajectories arriving in Tehran, highlighting that most of the back trajectories originated from the west of the study area. The Potential Source Contribution Function (PSCF) analysis could determine probable geographical locations and hotspot emission sources by calculating the probability fields resulting in high concentrations at the monitoring area. Detailed information about the PSCF analysis has been described elsewhere in Khuzestani et al. (2017). PSCF values representative of the potential hotspot areas could be calculated as follows:

Eq. $3 P S C F_{i j}=\frac{m_{i j}}{n_{i j}}$,

Where $n_{i j}$ is the total number of endpoints that pass through the grid cell $(i, j)$, and $m_{i j}$ is the number of endpoints related to the values that exceed the threshold criterion values in the same grid cell.

\section{Results And Discussions}

\section{1. $\mathrm{SO}_{2}$ variation profiles}

The general daily variation profile of $\mathrm{SO}_{2}$ in Tehran is displayed in Fig. 1a. Almost all monitoring areas showed two peaks for $\mathrm{SO}_{2}$ concentration when the first peak coincides in the morning rush hours and the second peak before midnight. The highest concentrations of $\mathrm{SO}_{2}$ in Tehran were experienced during 8:00 to 11:00 AM local time mainly due to the reduced Planetary Boundary Layer (PBL) heights and the increased traffic in Tehran. Greater PBL heights and the reduced traffic congestions during the afternoon period resulted in decreasing $\mathrm{SO}_{2}$ concentration to their minimum levels around 17:00 local time. With the increasing traffic activities nighttime, $\mathrm{SO}_{2}$ concentrations increased again to their highest levels at around 21:00. Due to the traffic rules in Tehran, heavy-duty diesel vehicles (HDDVs) are allowed to travel in the city from 10:00 PM to 6:00 AM local time (Taheri et al., 2019; Yeganeh et al., 2021). Therefore, we can expect that the first and second peaks could correspond to the increase in low-duty vehicles (LDVs) during the morning rush hours in addition to the HDDVs in parallel with reducing the PBL height, respectively. Considering that most $\mathrm{SO}_{2}$ in Tehran was generated from stationary sources, we can expect that the operation of thermal power plants and the available industries in the region would also largely contribute to higher $\mathrm{SO}_{2}$ concentrations in the area. In addition to the stationary sources, mobile sources would also contribute to the $\mathrm{SO}_{2}$ profiles in Tehran.

Diurnal variation patterns of $\mathrm{SO}_{2}$ were considerably different across the sampling sites in Tehran. Fig. 1d illustrates the time series of the diurnal variations of the concentrations of $\mathrm{SO}_{2}$ across all the sampling 
sites in Tehran. $\mathrm{SO}_{2}$ concentrations at R21 and SHAD areas increased at around 6:00 local time and moved to their highest levels at around 9:00 local time. During the afternoon, concentrations decreased again and reached their lowest values at around 17:00 local time. The concentration of $\mathrm{SO}_{2}$ during the nighttime period started to increase and reached its maximum at around 20:00 local time. R21 and SHAD areas are close to roadway traffic, and therefore their diurnal variation is greatly influenced by the traffic activities. $\mathrm{SO}_{2}$ concentration at these stations could also be affected by the northwestern emission sources in Tehran, and therefore, higher concentrations were experienced in these stations.

In the $\mathrm{R} 20$ area, we observed maximum $\mathrm{SO}_{2}$ concentrations at around 12:00 local time, and the concentrations started to decrease during the afternoon period. The area is nearby to Tehran industries and power plants; hence we expect that the operation of these industries would greatly influence $\mathrm{SO}_{2}$ concentration in this area. The magnitude of the diurnal variations of $\mathrm{SO}_{2}$ at AGD, TARB, and PIR areas was less pronounced compared with other monitoring areas in Tehran. These stations were located in residential areas of Tehran, and their variation profiles were less influenced by significant traffic and industrial operations in Tehran.

The seasonal variation patterns of $\mathrm{SO}_{2}$ concentration were presented in Fig. $2 \mathrm{~b}$ and e. In general, similar monthly variation patterns were observed for all the monitoring areas in Tehran. Relatively identical characteristics were observed with maximum concentrations experienced during winter (December, January, and February) and the lowest concentration during spring and summer (April-July) (Amini et al., 2014; Hassanzadeh et al., 2009). The lowest $\mathrm{SO}_{2}$ concentration was observed in April, coinciding with the new year holidays in Iran, leading to a sharp decline in transportation within the city. The increasing consumption of natural gas in Iran for residential heating purposes may correspond to higher $\mathrm{SO}_{2}$ concentrations during autumn and winter in Tehran (Nazari et al., 2012). In addition, as temperatures decrease and natural gas consumption increases in the domestic and commercial sectors, industries are forced to switch to alternative fuels with higher sulfur content, leading to more production and emissions of $\mathrm{SO}_{2}$ in Iran during autumn and winter. The decline in the planetary boundary layer (PBL) heights during the cold months cause the emitted $\mathrm{SO}_{2}$ to spread at a lower altitude, leading to a higher concentration of $\mathrm{SO}_{2}$.

The weekly variation profiles of $\mathrm{SO}_{2}$ were also illustrated in Fig. $1 \mathrm{c}$ and $\mathrm{f}$. Again, relatively similar variation patterns were observed for all our monitoring areas. AGD area had the lowest $\mathrm{SO}_{2}$ profile, while SHAD and R21 areas had the highest $\mathrm{SO}_{2}$ concentrations. In general, $\mathrm{SO}_{2}$ concentrations showed maximum levels on Mondays and Tuesdays (weekdays) and reached their minimum levels on Friday and Saturdays (Weekends in Iran).

\subsection{Bivariate Polar Plot (BPP) analysis}

The role of wind components on $\mathrm{SO}_{2}$ concentration variations for analysis of local and regional potential sources influences in Tehran are evaluated via the bivariate polar plots (BPPs) and illustrated in Fig. 2a-f. As displayed in Fig. 2, relatively similar profiles were observed in BPPs of all the monitoring areas in 
Tehran, demonstrating two primary potential hotspot sources for the $\mathrm{SO}_{2}$ emissions. The first identified hotspot area in the BPPs in all the monitoring areas showed high potential $\mathrm{SO}_{2}$ sources under low wind speed $(<5 \mathrm{~m} / \mathrm{s})$. Little wind direction dependence slightly expanded toward the southern directions in Tehran. We expect that these areas mainly dominate local emission sources originating from roadway traffic emissions and domestic heating under stagnant atmospheric conditions that help the potential accumulation and build-up of $\mathrm{SO}_{2}$ emissions from these local hotspot areas, resulting in higher $\mathrm{SO}_{2}$ concentrations (Hama et al., 2020).

In addition to the local hotspot areas illustrated in BPPs in all our monitoring areas, high potential $\mathrm{SO}_{2}$ emission sources were observed in northwestern areas indicating high potential $\mathrm{SO}_{2}$ emissions under high wind speeds $(\approx 10 \mathrm{~m} / \mathrm{s}$ ) originated from the air masses generated from the northwest. These high emitting plumes are emitted further away from the area showing characteristics of long-distance transport of $\mathrm{SO}_{2}$ from external hotspots located in the west and the northwestern regions of Tehran. Nearly all the monitoring areas in Tehran showed this characteristic indicating the high probability of existing persistent $\mathrm{SO}_{2}$ emissions from external locations in the northwestern areas of Tehran (Amini et al., 2014).

We have performed a non-parametric wind regression (NWR) approach of modeling the surfaces in BPPs using kernel smoothers that weigh $\mathrm{SO}_{2}$ on a surface according to the proximity to defined wind speed and direction intervals described by Henry et al. (2009). NWR approach provides the means to evaluate the stability of our analysis and confirm the consistency of the long-range transported hotspot areas illustrated in BPPs in all the monitoring areas. This analysis is similar to the BPP analysis described earlier, but it has significant advantages when sufficient data are not available to generate surfaces in some areas of BPPs. Fig. S4 in the supporting information shows the results of the NWR analysis of all the monitoring areas in Tehran. As illustrated in Fig. S4, our NWR approach shows relatively similar results to our BPP analysis. In addition to the local (central areas in the plot) and regional hotspots (located in the northwestern areas), high potential regional sources under high wind speeds $(\approx 10 \mathrm{~m} / \mathrm{s})$ originated from the southern areas were also observed in NWR analysis, illustrating probabilities of existence of high emitting $\mathrm{SO}_{2}$ sources located further away in southern parts of Tehran. The NWR approach generally showed similar results for our monitoring areas east of Tehran (e.g., AGD, PIR, and R20), pointing to northwestern areas as hotspot sources. However, other mentoring areas located in the western parts of Tehran mainly showed hotspot sources originating from the south/southwestern areas (Fig. S4). This again confirmed the availability of 2 main regionally transported emission sources of $\mathrm{SO}_{2}$ located further away in the west and southwest of the study area.

\section{3. $\mathrm{k}$-mean cluster analysis}

To further analyze the potential hotspot areas identified by BPP and NWR analysis, we performed k-mean clustering of the polar plots allowing various sources of $\mathrm{SO}_{2}$ in Tehran to be revealed in terms of the clusters. This method can characterize $\mathrm{SO}_{2}$ features into various homogenous clusters by minimizing the sum of the squared euclidean distances between the observed data and the cluster center. We performed 
k-mean cluster analysis for 2 - 10 cluster numbers, and the results are provided in Fig. S5 in the supporting information. Our analysis and comparison of the clustering database with the results obtained from BPP and NWR analysis showed that the 8-cluster solution could sufficiently define all the characteristics of $\mathrm{SO}_{2}$ variation features. The results from our 8-cluster analysis as well as the corresponding NWR and BPP plots for the general $\mathrm{SO}_{2}$ patterns in Tehran are illustrated in Fig. 3a-c. According to Fig. 3c, cluster 3 of our 8-cluster solution mostly represents the local sources; however, cluster 2 and cluster 7 could probably correspond to the external distant hotspot locations transported in Tehran from the southern and western areas, respectively, as observed in BPP and NWR plots (Fig. 3a and b). All other sources, rather than from clusters 3 and 6 , may also correspond to long-range external locations emitting $\mathrm{SO}_{2}$ in Tehran (will be discussed in the following section). However, our postprocessing of $\mathrm{SO}_{2}$ profiles in all of the monitoring stations in Tehran (Fig. 4) confirmed that clusters 2, 3, and 7 had the highest contributions compared with other clusters observed in Fig. 3c. As described earlier, cluster 3 mainly corresponded to local $\mathrm{SO}_{2}$ emissions generated from the roadway traffic emissions; however, clusters 2 and 7 mainly corresponded to distant external hotspot emission sources located further away from the region.

The constructed diurnal variation profiles of cluster 3 (Fig. S6) agreed reasonably well with the variations of general $\mathrm{SO}_{2}$ diurnal profiles in Tehran observed in Fig. 1, implying that cluster 3 could remarkably illustrate the characteristics of local emission sources in all the monitoring stations in Tehran. The diurnal variation profiles in clusters 2 and 7 were not so profound and did not agree well with that observed for cluster 3 and the general $\mathrm{SO}_{2}$ profiles implying that other emission sources rather than local traffic emissions could also impact $\mathrm{SO}_{2}$ profiles in other clusters in Tehran.

Our observations showed that large $\mathrm{SO}_{2}$ emissions resulting from the consumption of heavy fuel oils in thermal power plants located in western and northwestern areas of Tehran could greatly affect $\mathrm{SO}_{2}$ emissions in Tehran (Amini et al., 2014). Fig. S7 and S8 in the supporting information illustrated the existence of large $\mathrm{SO}_{2}$ plumes mainly from thermal power plants located in the west and southern areas of Tehran. This agreed reasonably well with our BPP, NWR, and cluster analysis, implying potential hotspot sources in the western areas (cluster 7). In addition, large petroleum and fuel oil industries and thermal power plants located in the southern parts of Tehran are also shown with high $\mathrm{SO}_{2}$ emissions from cluster 2 hotspot areas. Lack of flue gas desulfurization and other control measures to minimize $\mathrm{SO}_{2}$ plumes from thermal power plants in the region resulted in uncontrolled and significant $\mathrm{SO}_{2}$ emissions from external hotspot locations transported in the city (Nazari et al., 2012).

\subsection{Pairwise polar correlation analysis}

Pairwise regression of $\mathrm{SO}_{2}$ concentrations for all the individual sites was compared to evaluate the impacts from local/regional sources of all $\mathrm{SO}_{2}$ emissions across all the monitoring areas in Tehran. $\mathrm{A}$ linear fit was integrated with each regression analysis to define the slope, slope uncertainty, intercept, intercept uncertainty, and $\mathrm{R}^{2}$ value (Table S3). To statistically evaluate the effects of the local/regional 
sources between the monitoring areas in Tehran, the slopes and intercepts of the regression lines generated from each pair were analyzed to see if they are statistically significant by deciding if 1 minus the slope is larger than two times the uncertainty, or the absolute intercept value is larger than two times of intercept uncertainty (Khuzestani et al., 2017; McGinnis et al., 2014). Detailed information on the pairwise regression analysis is provided in Table $S 2$ in the supporting information. Most of our location pairs showed the influence of local emissions by indicating statistically significant slopes and intercepts. However, some of our pairs illustrated the regional transport of $\mathrm{SO}_{2}$ emissions in Tehran.

To visually illustrate the effects of regional hotspot sources in the region, we consider illustrating the Pearson correlations coefficients $(r)$ derived from the pairwise regression analysis between each pair to vary against wind components. The combination of pairwise correlation analysis with polar plots could provide significant insight into the source apportionment information (Grange et al., 2016). This analysis involves weighting $r$ values obtained from pairwise regression analysis by wind speed/direction bins. A Gaussian kernel model used for polar plots was implemented to guarantee that maximum data is used in every wind speed-direction interval (Grange et al., 2016). The results of our pairwise polar correlation analysis are provided in Fig. 5. Very high correlation coefficients between all the pairwise cases were experienced under higher wind speeds and different wind directions. Significant sources of $\mathrm{SO}_{2}$ in Tehran were observed from external emission sources, again illustrating the effects of long-range transport of $\mathrm{SO}_{2}$ in Tehran. Comparison analysis of the cases depending on the location, characteristics, and the distance between our monitoring areas could reveal different hotspot areas for $\mathrm{SO}_{2}$ emission sources. However, regardless of the pairwise correlation analysis, all the cases were able to detect relatively similar source origins corresponding to large long-range transport of $\mathrm{SO}_{2}$ emissions from external locations in the west/northwestern areas (cluster 4 and 7), north/northeast areas (cluster 5 and 8 ) and southern/southwest areas (cluster 1 and 2). This agreed perfectly with the results from our k-mean clustering analysis, BPP, NWR, and finally PSCF analysis, confirming that all the clusters (except clusters 3 and 6) could correspond to significant regional long-range transport of $\mathrm{SO}_{2}$ emission from external locations in Tehran. None of our pairwise correlation polar plots detected low wind speed central areas (clusters 3 and 6), implying significant impacts of long-range transport of $\mathrm{SO}_{2}$ from several external high emitting sources located further away from Tehran.

\subsection{Effects of regional transport}

The TROPOMI data analysis illustrated an extensive line of $\mathrm{SO}_{2}$ emission sources covering western parts of the country with main hotspots related to Iraq, Kuwait and Saudi Arabia. Some other high emitting sources located in Turkey and Uzbekistan were also observed in TROPOMI data analysis (Fig. 6). PSCF analysis results for all the monitoring sites in Tehran are also shown in Fig. 7. Our PSCF analysis showed a high probability of emission sources in the Iraq and Kurdistan area in the west, Saudi Arabia in the southwest, and adjacent parts in the north, east and southeastern areas. Interestingly, our further observations of these hotspot locations showed that the observed source regions agreed perfectly with the results of the TROPOMI SO${ }_{2}$ properties of the spatial distributions of columnar $\mathrm{SO}_{2}$ over Iran and its 
surrounding region for the whole year of 2020 (Fig. 6) as well as the NASA global $\mathrm{SO}_{2}$ emission source catalog as identified power plants and oil and gas refineries with high $\mathrm{SO}_{2}$ emissions in the region. This was also consistent with the results of our BPP, k-mean clustering, and polar correlation analysis, further demonstrating the evidence of large-scale transport of $\mathrm{SO}_{2}$ from distant hotspots located away from Tehran. This suggests that high $\mathrm{SO}_{2}$ emissions from the industries in Iran's neighboring countries (i.e., Iraq and Saudi Arabia as identified in our PSCF and TROPOMI SO ${ }_{2}$ data analysis) in addition to the high emitting $\mathrm{SO}_{2}$ sources located in surrounding areas of Tehran would significantly affect $\mathrm{SO}_{2}$ emissions in our monitoring sites.

\section{Conclusions}

$\mathrm{SO}_{2}$ emission is considered a critical air quality concern in Tehran, often causing complex atmospheric pollution situations in that middle eastern megacity. Herein, comprehensive and quantitative studies on $\mathrm{SO}_{2}$ emission production and their local and regional hotspot sources in Tehran were evaluated using various analysis methods, including bivariate polar plots, k-mean clustering, pairwise correlation analysis, potential source contribution function as well as the remote sensing TROPOMI $\mathrm{SO}_{2}$ spatial distribution. Our analysis of different monitoring areas in Tehran confirmed that local sources originated from roadway traffic emissions, and domestic heating only contributes a minor fraction (20\%) to the total $\mathrm{SO}_{2}$ productions in Tehran. Approximately $80 \%$ of the total $\mathrm{SO}_{2}$ emissions were transported in Tehran throughout long-range transport from different external hotspot areas located further away from Tehran. Our analysis with BPP, NWR, pairwise polar correlation, and PSCF analysis confirmed that similar source origins corresponding to large long-range transport of $\mathrm{SO}_{2}$ emissions from external locations related to Iraq and Kurdistan area in the west, Saudi Arabia in the southwest, and adjacent areas in north, east and southeastern areas. Our further observations of those hotspot areas with remote sensing TROPOMI SO data processing illustrated large $\mathrm{SO}_{2}$ emissions resulting from the consumption of heavy fuel oils in thermal power plants located in the west and southwestern areas of Tehran. Lack of proper control measures, including flue gas desulfurization process to control $\mathrm{SO}_{2}$ emissions from thermal power plants and oil/gas refineries, has resulted in significant $\mathrm{SO}_{2}$ emissions in Tehran from external hotspot locations transported in the city. The variability in contributions of local/regional emission sources has implications for potential control strategies that could reduce public exposures. Our analysis showed that limiting the high emitting vehicles and other regulatory strategies for controlling traffic-related emissions could be beneficial in reducing the exposure to many pollutants in Tehran. However, such strategies would be less effective for reducing the exposure to $\mathrm{SO}_{2}$ because of the significant contribution of longrange $\mathrm{SO}_{2}$ transport from external locations affecting the city.

\section{Declarations}

\section{Acknowledgements}


The authors thank the CEO and staff of the Tehran Air Quality Control Company for providing the $\mathrm{SO}_{2}$ concentration and other relevant data sets.

\section{References}

1. Amini H, Taghavi-Shahri SM, Henderson SB, Naddafi K, Nabizadeh R, Yunesian M. Land use regression models to estimate the annual and seasonal spatial variability of sulfur dioxide and particulate matter in Tehran, Iran. Science of the total environment 2014; 488: 343-353.

2. Bayat R, Ashrafi K, Motlagh MS, Hassanvand MS, Daroudi R, Fink G, et al. Health impact and related cost of ambient air pollution in Tehran. Environmental research 2019; 176: 108547.

3. Carslaw DC, Beevers SD. Characterising and understanding emission sources using bivariate polar plots and k-means clustering. Environmental modelling \& software 2013; 40: 325-329.

4. Carslaw DC, Beevers SD, Ropkins K, Bell MC. Detecting and quantifying aircraft and other on-airport contributions to ambient nitrogen oxides in the vicinity of a large international airport. Atmospheric Environment 2006; 40: 5424-5434.

5. Dahiya S, Myllyvirta L. Global SO2 emission hotspot database, Ranking of the world's worst sources of SO2 pollution. Greenpeace Environment Trust 2019: 1-13.

6. Esmaeilirad S, Lai A, Abbaszade G, Schnelle-Kreis J, Zimmermann R, Uzu G, et al. Source apportionment of fine particulate matter in a Middle Eastern Metropolis, Tehran-Iran, using PMF with organic and inorganic markers. Science of the Total Environment 2020; 705: 135330.

7. Ghadiri Z, Rashidi Y, Broomandi P. Evaluation Euro IV of effectiveness in transportation systems of Tehran on air quality: Application of IVE model. Pollution 2017; 3: 639-653.

8. Grange SK, Lewis AC, Carslaw DC. Source apportionment advances using polar plots of bivariate correlation and regression statistics. Atmospheric Environment 2016; 145: 128-134.

9. Hama SM, Kumar P, Harrison RM, Bloss WJ, Khare M, Mishra S, et al. Four-year assessment of ambient particulate matter and trace gases in the Delhi-NCR region of India. Sustainable Cities and Society 2020; 54: 102003.

10. Hassanzadeh S, Hosseinibalam F, Alizadeh R. Statistical models and time series forecasting of sulfur dioxide: a case study Tehran. Environmental monitoring and assessment 2009; 155: 149-155.

11. Henry R, Norris GA, Vedantham R, Turner JR. Source region identification using kernel smoothing. Environmental science \& technology 2009; 43: 4090-4097.

12. Khuzestani RB, Schauer J, Wei Y, Zhang L, Cai T, Zhang Y, et al. Quantification of the sources of longrange transport of PM2.5 pollution in the Ordos region, Inner Mongolia, China. Environmental Pollution 2017; 229: 1019-1031.

13. McGinnis JE, Heo J, Olson MR, Rutter AP, Schauer JJ. Understanding the sources and composition of the incremental excess of fine particles across multiple sampling locations in one air shed. Journal of Environmental Sciences 2014; 26: 818-826. 
14. Nazari S, Shahhoseini O, Sohrabi-Kashani A, Davari S, Sahabi H, Rezaeian A. SO2 pollution of heavy oil-fired steam power plants in Iran. Energy policy 2012; 43: 456-465.

15. Shahbazi $\mathrm{H}$, Hassani A, Hosseini V. Evaluation of Tehran clean air action plan using emission inventory approach. Urban Climate 2019; 27: 446-456.

16. Taheri A, Aliasghari P, Hosseini V. Black carbon and PM2. 5 monitoring campaign on the roadside and residential urban background sites in the city of Tehran. Atmospheric Environment 2019; 218: 116928.

17. Westmoreland EJ, Carslaw N, Carslaw DC, Gillah A, Bates E. Analysis of air quality within a street canyon using statistical and dispersion modelling techniques. Atmospheric Environment 2007; 41: 9195-9205.

18. Yeganeh B, Khuzestani RB, Taheri A, Schauer JJ. Temporal trends in the spatial-scale contributions to black carbon in a Middle Eastern megacity. Science of The Total Environment 2021: 148364.

19. Zhong Q, Shen H, Yun X, Chen Y, Ren Ya, Xu H, et al. Global sulfur dioxide emissions and the driving forces. Environmental science \& technology 2020; 54: 6508-6517.

\section{Figures}
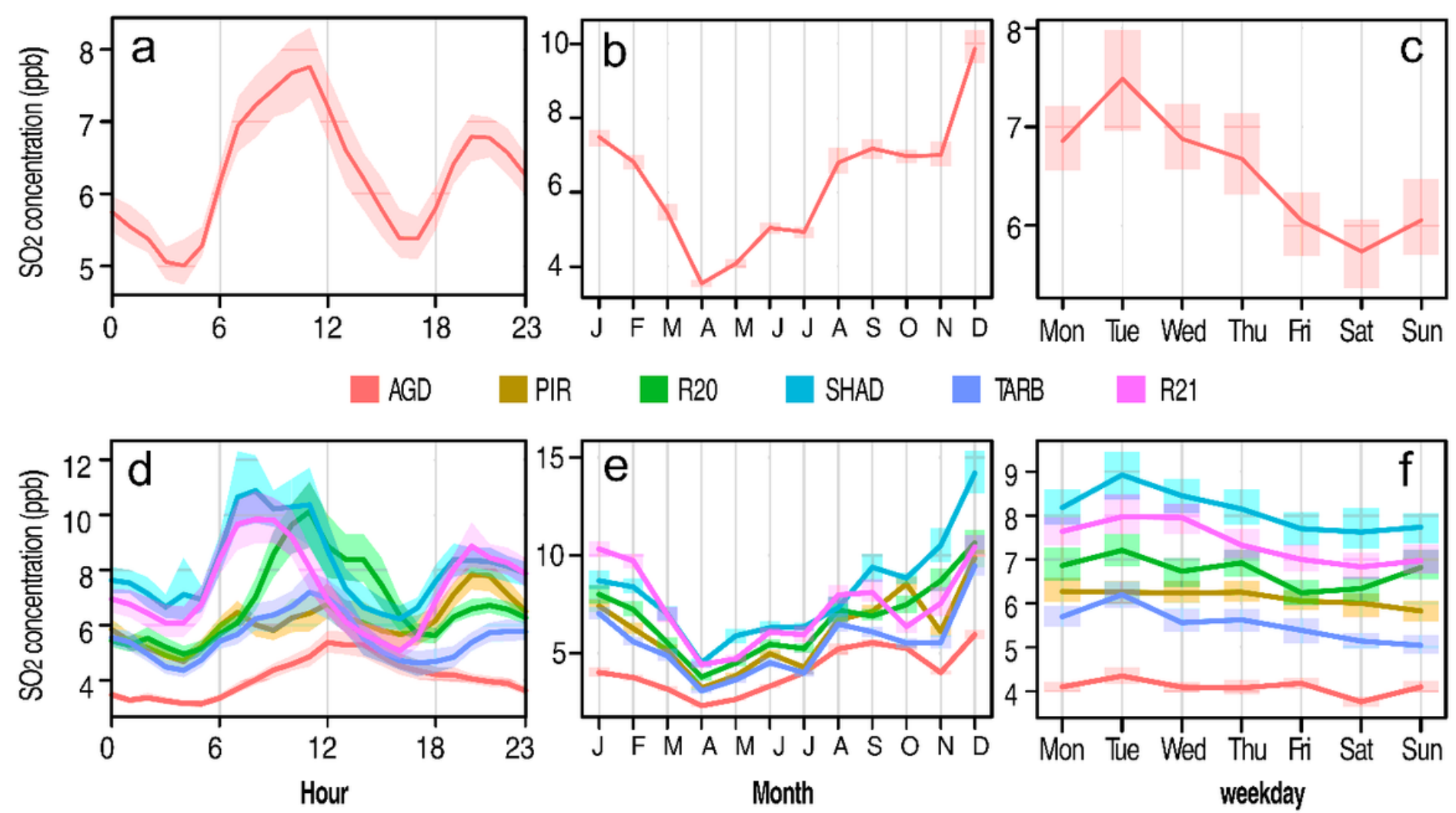

PIR
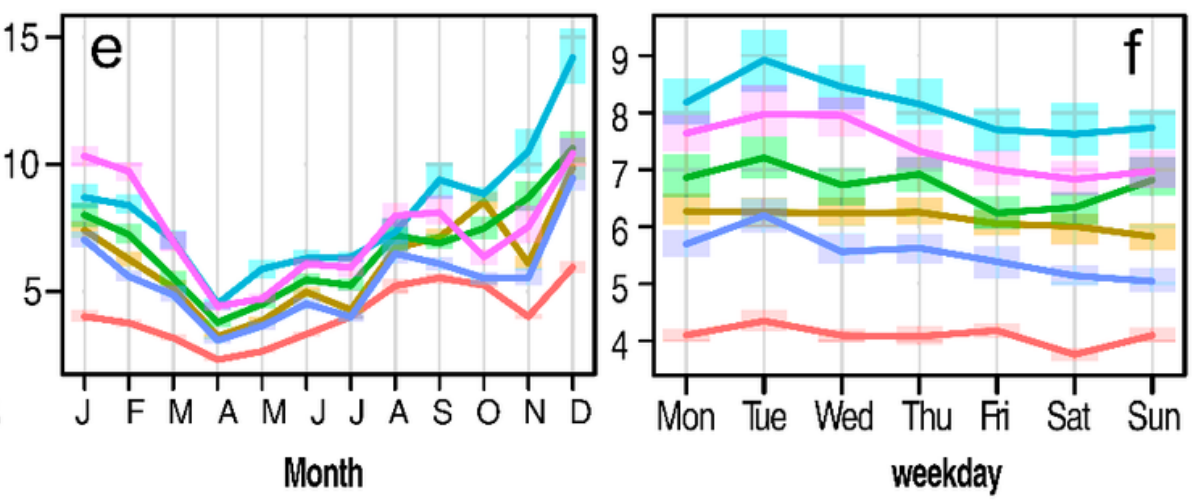
Figure 1

Hourly, monthly and weekly concentration profiles of $\mathrm{SO}_{2}$ in Tehran. $a, b$, and c panels represent concentrations profiles for the average $\mathrm{SO}_{2}$ concentrations, while $d, e$, and $f$ panels represent the variation patterns for each specific monitoring site in Tehran
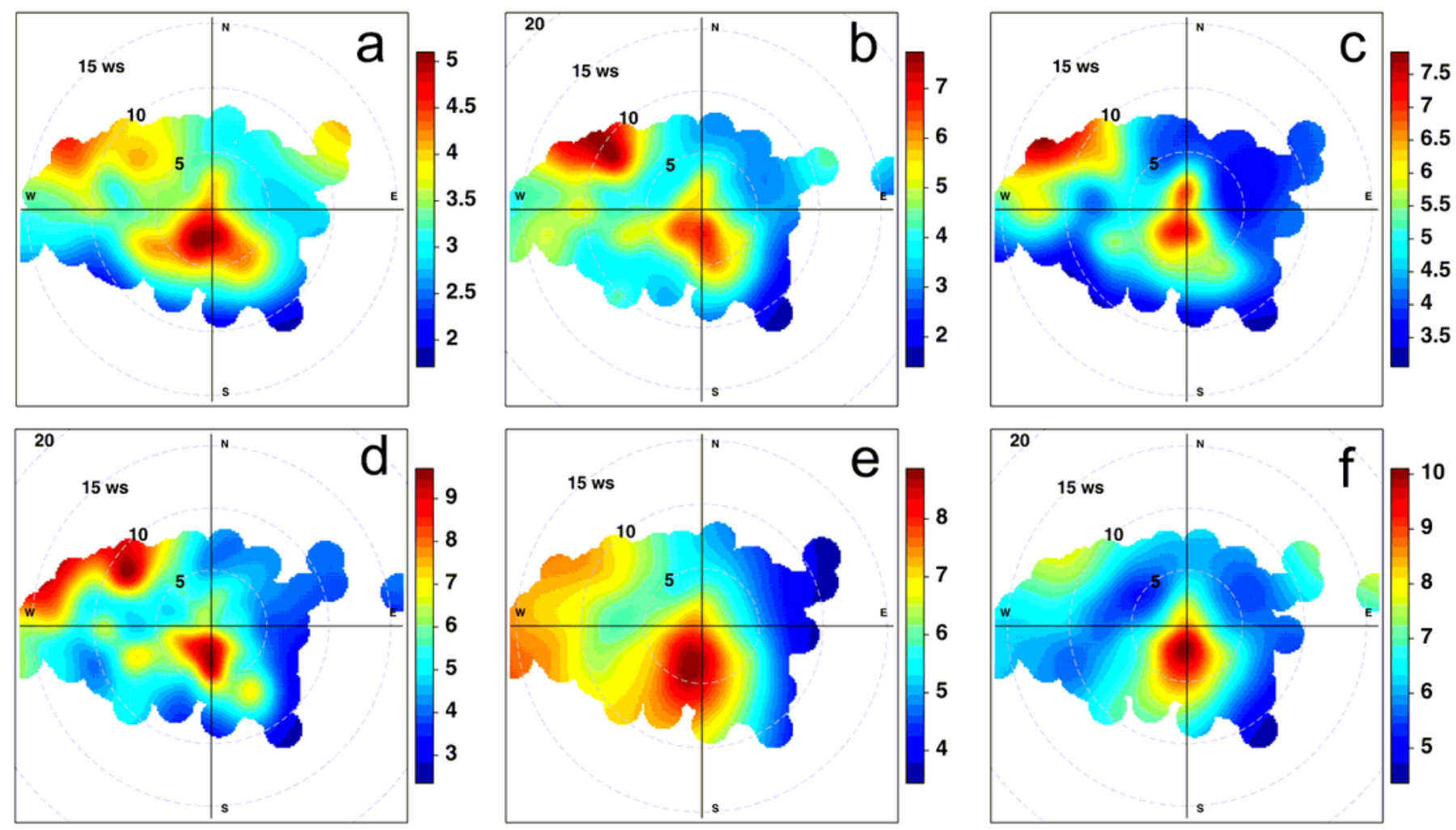

Figure 2

Bivariate concentration polar plots (BPP) analysis of $\mathrm{SO}_{2}$ in each monitoring area in Tehran. a) $\left.A G D, b\right)$ $T A R B$, c) PIR, d) R20, e) R21, and f) SHAD. 


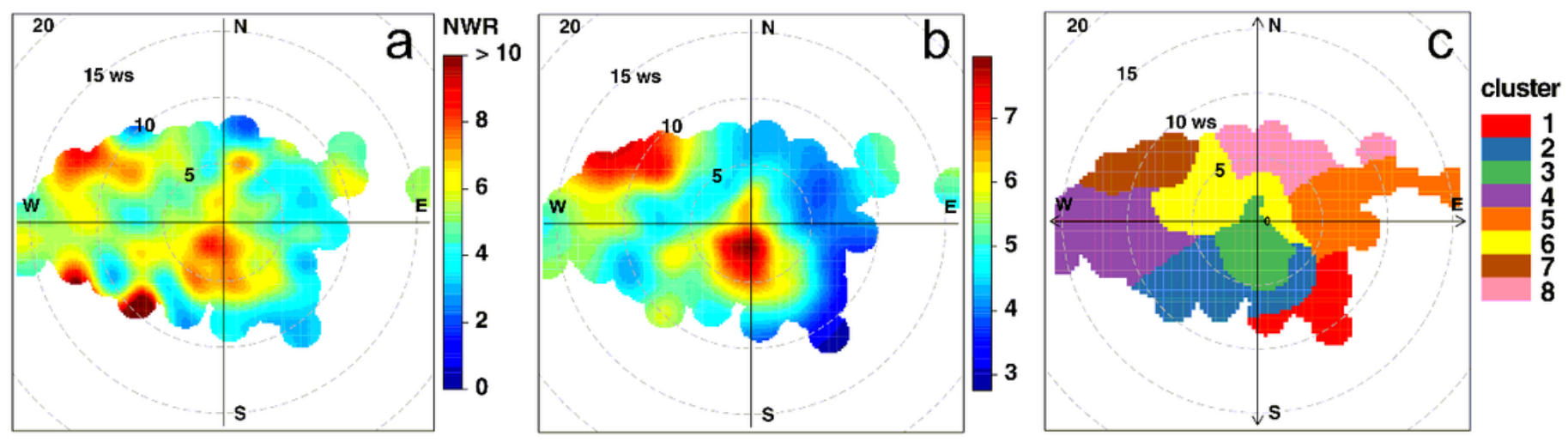

Figure 3

NWR, BPP and $k$-mean cluster analysis of the general $\mathrm{SO}_{2}$ profiles in Tehran. a) NWR analysis, b) BPP analysis, and c) 8-cluster solution analysis. 


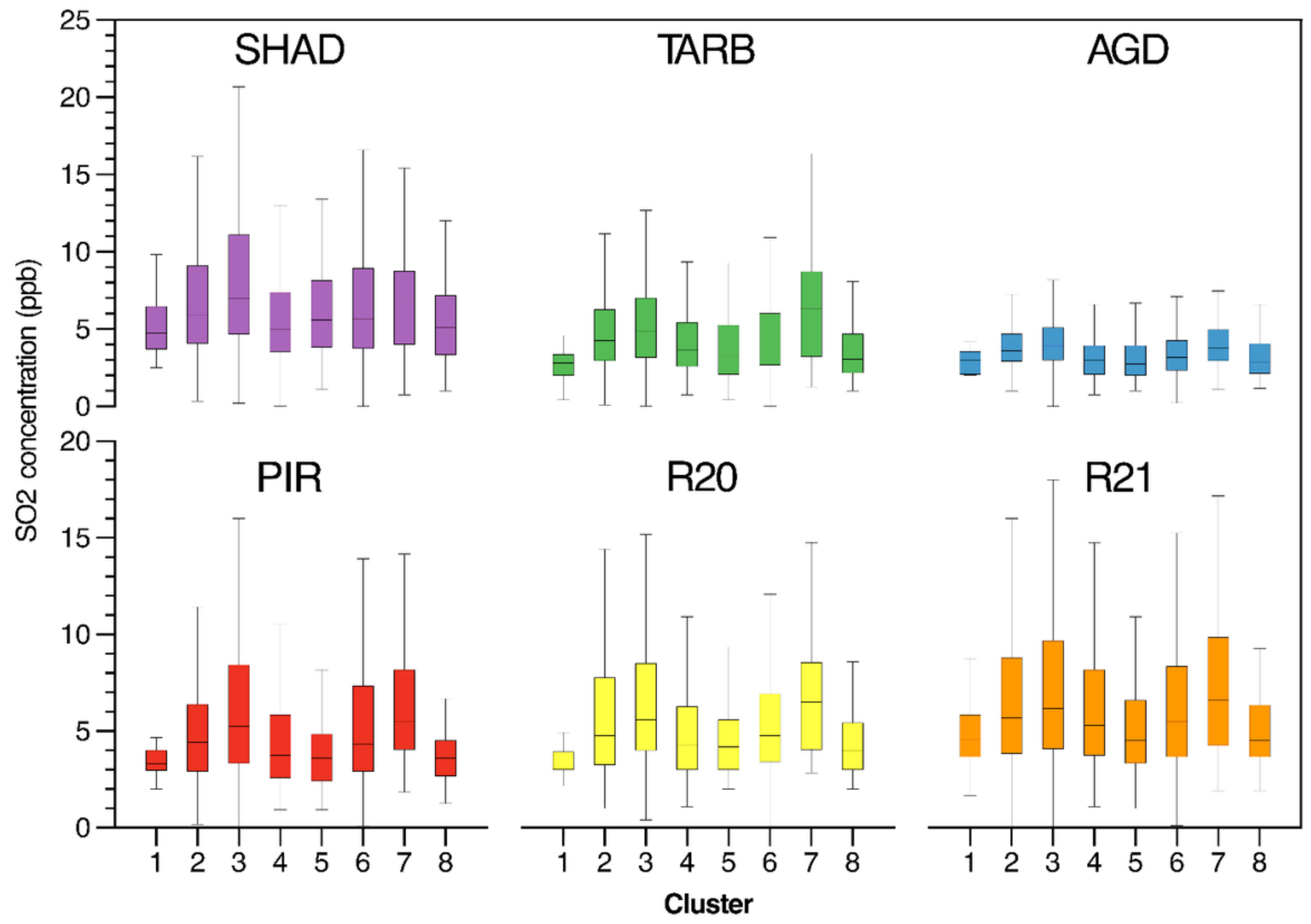

Figure 4

$\mathrm{SO}_{2}$ variation profiles for each monitoring site separated by clusters observed in our 8-cluster solution 

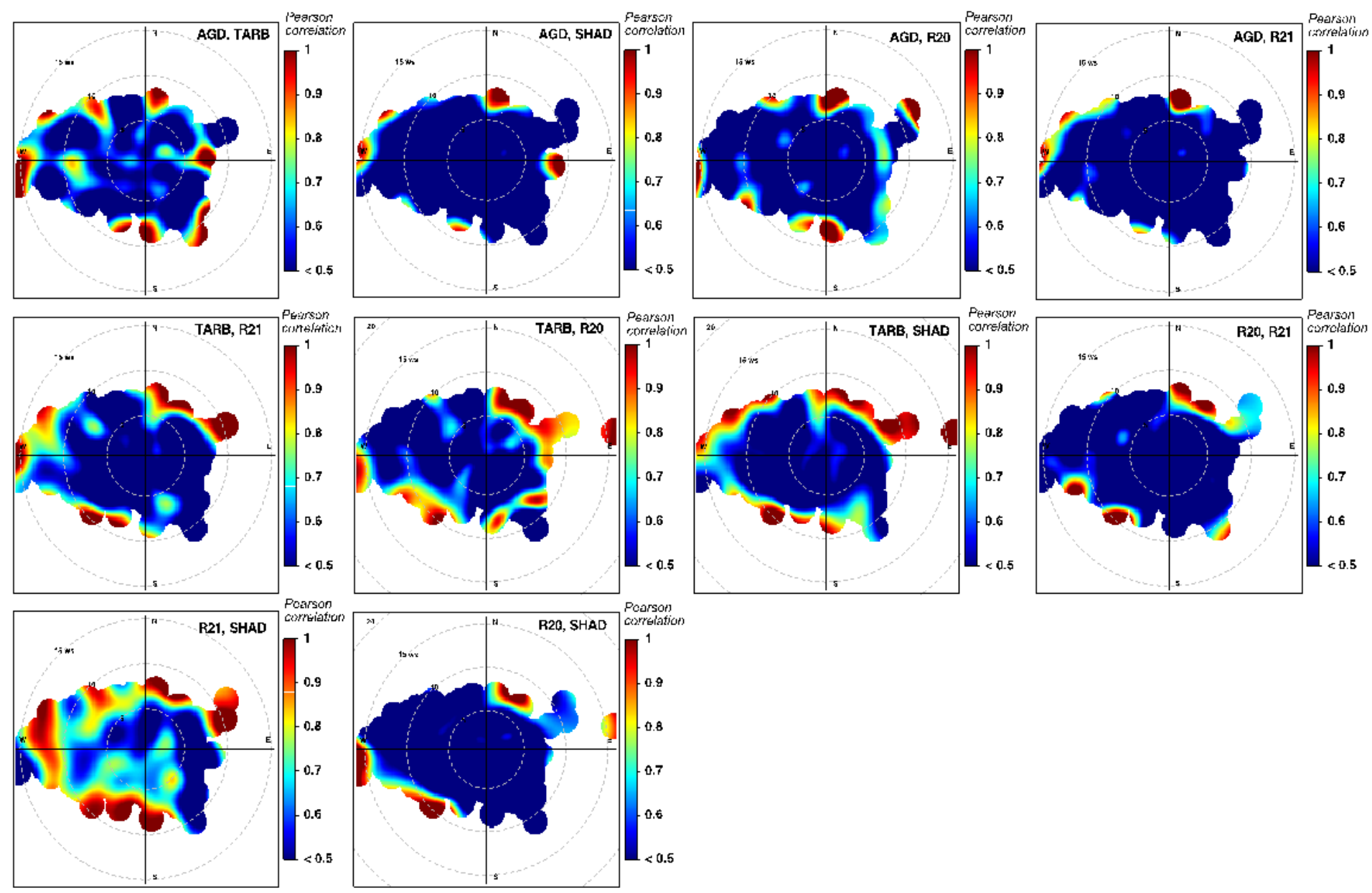

Figure 5

Pairwise polar correlation plots obtained from pairwise regression of $\mathrm{SO}_{2}$ concentrations for all the individual sites 


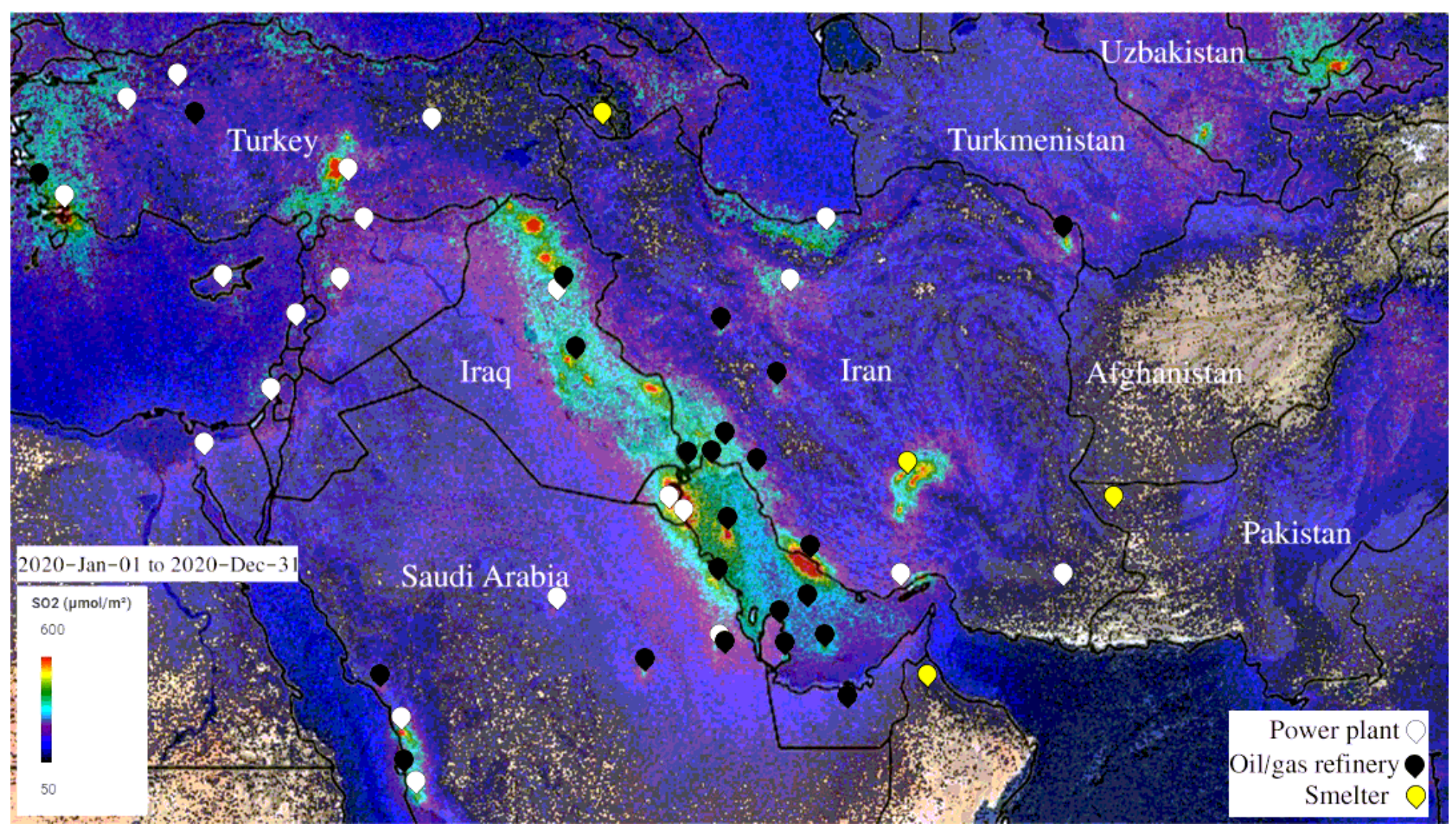

Figure 6

TROPOMI SO $\mathrm{S}_{2}$ properties of the spatial distributions of columnar $\mathrm{SO} 2$ over Iran and its surrounding region for the whole year of 2020 


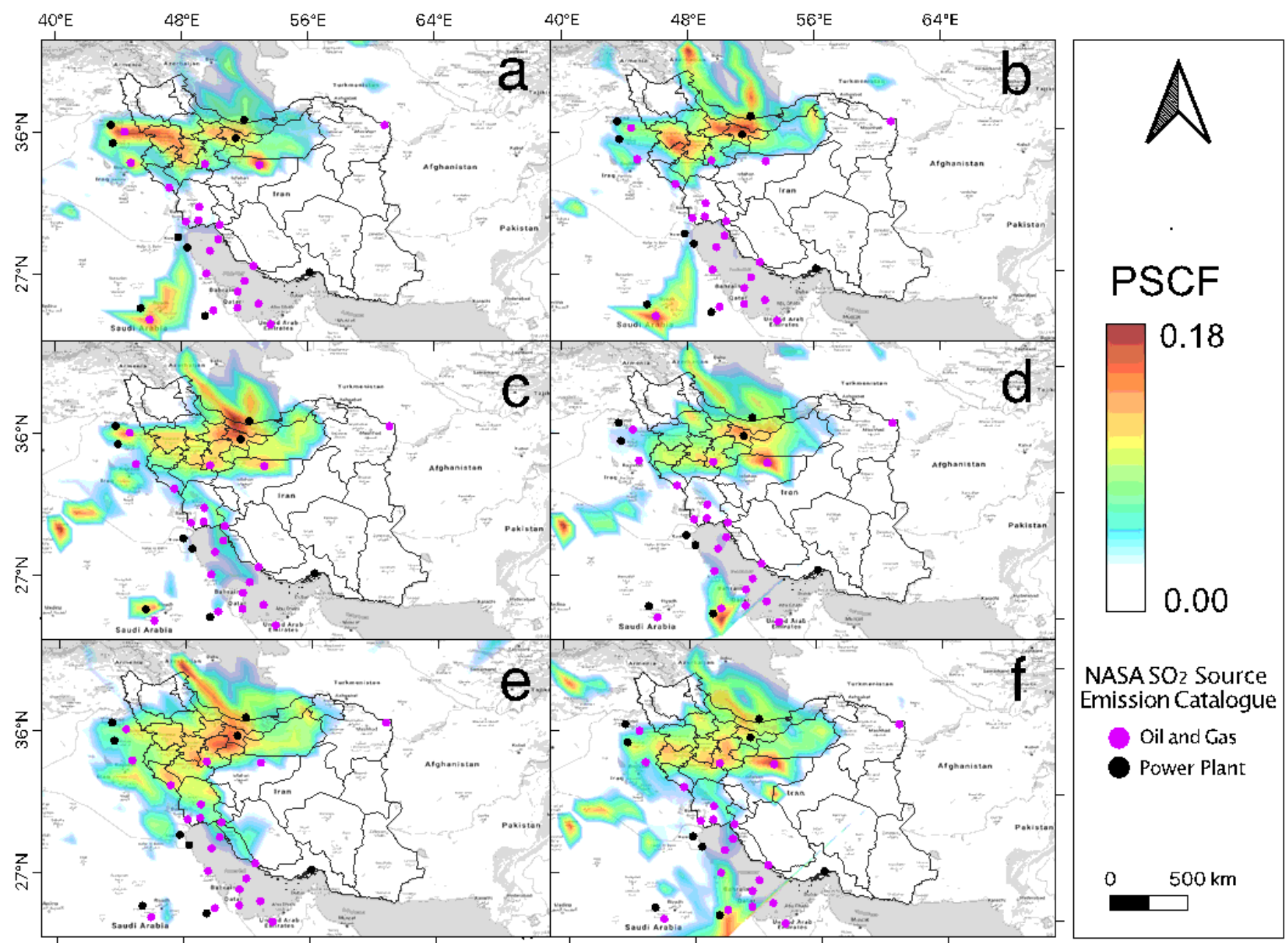

Figure 7

Smoothed PSCF map of $\mathrm{SO}_{2}$ emission hotspots in Tehran along with the observed $\mathrm{NASA} \mathrm{OMI} \mathrm{SO} \mathrm{S}_{2}$ source emission catalogue identifying high emitting power plants and oil and gas refineries around the region. a) $A G D, b) P I R$, c) TARB, d) SHAD, e) R20, f) R21.

\section{Supplementary Files}

This is a list of supplementary files associated with this preprint. Click to download.

- Slv8.docx 